\title{
Platelet Count, ADAMTS13 Activity, von Willebrand Factor Level and Survival in Patients with Colorectal Cancer: 5-Year Follow-up Study
}

\author{
Nóra Garam ${ }^{1, *}$ Éva Maláti ${ }^{1, *}$ György Sinkovits ${ }^{1}$ Tímea Gombos ${ }^{1}$ Attila Szederjesi ${ }^{2}$ \\ Loránd Barabás $^{3}$ László Gráf ${ }^{1}$ Judit Kocsis ${ }^{1}$ Zoltán Prohászka ${ }^{1}$
}

\footnotetext{
${ }^{1}$ Research Laboratory, Illrd Department of Internal Medicine, MTA-SE Research Group of Immunology and Hematology, Hungarian Academy of Sciences and Semmelweis University, Budapest, Hungary

2 Department of Hematology and Stem Cell Transplantation, United St. Istvan and St. Laszlo Hospital, Budapest, Hungary

${ }^{3}$ 2nd Department of Surgery, Faculty of Medicine, Semmelweis University, Budapest, Hungary
}

\begin{abstract}
Address for correspondence Nóra Garam, MD, Research Laboratory, IIIrd Department of Internal Medicine, MTA-SE Research Group of Immunology and Hematology, Hungarian Academy of Sciences and Semmelweis University, H-1125 Budapest, Kútvölgyi st. 4, Hungary (e-mail: norigaram@gmail.com).
\end{abstract}

\begin{abstract}
Keywords

- ADAMTS13

- platelet

- von Willebrand factor

- mortality

- colorectal cancer

Distant metastasis is a major cause of colorectal cancer-related death, but the mechanism of tumour progression is not fully understood. There is growing evidence of an interaction between tumour cells and platelets which may influence tumour progression and metastasis formation. Quality and quantity of von Willebrand factor may regulate the interaction between tumour cells and platelets. Our aim was to measure the platelet count, von Willebrand factor antigen (VWF:Ag) levels and ADAMTS13 activity in a large $(n=232)$ cohort of colorectal cancer patients and to examine their relationships with the stage of the disease and 5-year survival without thrombotic complications using multivariable models. Significantly higher platelet counts $(p=0.005)$, VWF:Ag levels $(p=0.008)$ and decreased ADAMTS13 activity $(p=0.006)$ were observed in patients with metastatic disease. Results of the KaplanMeier analysis showed that lower platelet counts $(p<0.0001)$, lower VWF:Ag $(p=0.0008)$ levels and higher ADAMTS13 activity $(p<0.0001)$ were associated with better event-free survival. Finally, to investigate the association between overall event-free survival and the three study variables, multivariate Cox proportional hazard models were generated. All models were adjusted for age, gender and disease stage. Platelet count, ADAMTS13 activity or VWF:Ag level were incorporated and all of these variables turned out to be age-, gender- and stage-independent predictors of mortality (all hazard ratio $>1.7, p<0.05$ ). In summary, this is the first observational study reporting association between higher mortality or thrombotic complications and increased platelet count, increased VWF:Ag levels and decreased ADAMTS13 activity in colorectal cancer.
\end{abstract}

* Nóra Garam and Éva Maláti have equally contributed to this study.

received

August 10, 2017

accepted after revision

October 13, 2017
Copyright () 2018 Schattauer

DOI https://doi.org/ 10.1160/TH17-07-0548. ISSN 0340-6245. 


\section{Introduction}

Colorectal cancer is one of the leading causes of mortality all over the world. Based on the WHO World Cancer Report 2014, colorectal cancer is the third most prevalent cancer type in men and the second in women. It is the third most common cause of cancer-related mortality in men and fourth in women.

The mortality decreased in the last decades due to colorectal screening and therapeutic improvements, ${ }^{1}$ but in spite of that the presence of distant metastasis is a major cause of colorectal cancer-related death. ${ }^{2}$ The survival of patients with metastatic colorectal cancer is even worse than the localized form of the disease. ${ }^{3}$

It is important to find new reliable markers that can predict the prognosis of colorectal cancer patients.

The contribution of platelets in metastasis formation has been known for a long time. First, it was observed in the mid19th century that venous thromboembolism and superficial thrombophlebitis occur together with malignancy. ${ }^{4}$ There is growing evidence of an interaction between tumour cells and platelets, which promote tumour progression. ${ }^{5}$ On the one hand, tumour cells can induce platelet activation; on the other hand, platelets can also promote tumour growth and metastasis formation with various mechanisms reviewed recently. ${ }^{4}$ Multiple studies investigated the role of platelets in several cancer types, such as ovarian, ${ }^{6}$ lung ${ }^{7,8}$ and colorectal cancer. $^{9-13}$ Preoperative thrombocytosis was associated with poor survival in surgically resected colorectal cancer patients ${ }^{14}$ and was identified as independent predictor of survival. ${ }^{11,12}$ Paraneoplastic thrombocytosis was shown to be associated with decreased median time to disease progression in ovarian cancer patients. ${ }^{15}$

The interaction between tumour cells and platelets could be mediated by multiple factors, ${ }^{4,5}$ among which the von Willebrand factor (VWF) belongs to the most widely studied group. This multimeric glycoprotein is expressed in and released from platelets and endothelial cells, and can bind to platelet surface receptors facilitating the formation of the platelet thrombus. In clinical studies, an increased plasma level of VWF antigen (VWF:Ag) was observed in various cancer types such as ovarian, bladder, gastric and colon cancer, and increased levels were associated with poor prognosis. ${ }^{16,17}$

The most adhesive form of the VWF is the ultra-large VWF, which is released from the endothelial cells. Under physiological circumstances, it is cleaved by ADAMTS13 (a disintegrin and metalloproteinase with thrombospondin type-I repeats 13) protease into the multimer form in a sheardependent manner. ${ }^{18}$ Significantly decreased ADAMTS13 serum levels were found in patients with localized or disseminated tumours compared to healthy individuals. ${ }^{19}$ In another study, an increased amount of ultra-large VWF multimers and decreased ADAMTS13 activity were observed in blood samples of patients with malignancies. ${ }^{20,21}$

Only few studies were performed in connection with VWF: Ag level and ADAMTS13 activity in tumours. In patients with disseminated metastasis, a reduced plasma level of ADAMTS13 was measured with the presence of unusually large VWF multimers. ${ }^{22}$ Similar to this observation, a lower mean plasma level of ADAMTS13 was found in disseminated malignancies compared with the localized form. The ADAMTS13 activity and VWF:Ag level correlate inversely. ${ }^{19}$ Although the epidemiological evidence supporting the potential association of increased platelet count and VWF:Ag level, or decreased ADAMTS13 activity with poor survival in cancer patients, is accumulating, none of the large studies evaluated these factors simultaneously in colorectal cancer patients. Therefore, our aim was to measure the platelet count, VWF:Ag level and ADAMTS13 activity and examine its association with the presence of ultra-large VWF in a large $(n=232)$ cohort of colorectal cancer patients, and to examine their relationships with the presence of metastatic disease and 5-year survival without thrombotic complications using multivariable models. Because the higher prothrombotic risk is associated with increased platelet count and VWF:Ag level, or decreased ADAMTS13 activity, we also compared these parameters in relation to the thrombotic complications during the disease.

\section{Materials and Methods}

\section{Patients}

A total of 232 patients diagnosed with colorectal cancer were enrolled in the study between January 2011 and June 2013 in the Oncology Department of the 3rd Department of Internal Medicine, Semmelweis University. The study was approved by the Medical Research Council Scientific and Research Committee and was performed at the 3rd Department of Internal Medicine, Semmelweis University, based on a study protocol approved by the highest Ethical Committee of Hungary. Patients' consent was obtained consecutively after the confirmation of colorectal cancer with any stage, which was based on the TNM staging system. Blood samples were taken after 6 hours of fasting between 8 and 10 Am by antecubital venipuncture into native, EDTA, or sodium citrate-anticoagulated tubes before starting any anticancer therapy. The samples were aliquoted and stored at $-70^{\circ} \mathrm{C}$ for further analysis. Clinical data were collected. Baseline demographics and clinical characteristics of patients are summarized in -Table 1. The mean age of patients was 68.5 years, with a male:female ratio of 138:94. After diagnosis and adequate surgery, patients were treated and followed up at the Oncology Department according to the stage of their disease and to the relevant guidelines. Patients with rectal cancer received radiochemotherapy before definitive surgery from cT3 or $\mathrm{N}+$ disease. Twenty-two patients who had irresectable and/or metastatic disease received upfront primary systemic treatment without definitive surgery. The data of thrombotic complications, which were defined as occurrence of deep vein thrombosis, pulmonary embolism, arterial occlusion with infarction and superficial thrombophlebitis, ${ }^{23}$ were collected from the disease onset until the last visit (median follow-up: 42.36 months). Patients were followed up for 5 years (median: 46.42 months) in the same institution and survival and thrombotic data were recorded and defined as events. Most of the patients died due to progressive malignant disease. Since there was no autopsy in the majority of cases, the cause of death, whether it was cardiovascular or cancer related, was not differentiated; therefore, mortality was defined as 
Table 1 Clinical and laboratory characteristics of patients with colorectal cancer

\begin{tabular}{|c|c|c|c|c|}
\hline & $\begin{array}{l}\text { Patients who had an } \\
\text { event within } 5 y^{a}\end{array}$ & $\begin{array}{l}\text { Patient who } \\
\text { had no event }\end{array}$ & $p$-Value ${ }^{c}$ & All patients \\
\hline Number of patients & 105 & 127 & - & 232 \\
\hline Males/Females & $64 / 41$ & $74 / 53$ & 0.607 & $138 / 94$ \\
\hline Age at diagnosis, $y$ & $70.3(65-76.2)^{b}$ & $65.2(58-72)$ & $<0.0001$ & $68.5(59.6-74.1)$ \\
\hline Platelet G/L & $309(240-413)$ & $271(217-326)$ & 0.013 & $286(224.5-364.50)$ \\
\hline ADAMTS activity, \% & $71.17(59.33-88.45)$ & 83.95 (70.71-104.66) & $<0.0001$ & 78.40 (65.02-96.79) \\
\hline VWF antigen, ng/mL & $665.74(369.75-1,433.4)$ & 414.75 (254.37-764.54) & 0.001 & $521.83(286.10-1,067.97)$ \\
\hline $\begin{array}{l}\text { Carcinoembryonic } \\
\text { antigen, } \mu \mathrm{g} / \mathrm{L}\end{array}$ & $4.45(2-55)$ & $1.8(1-3.4)$ & $<0.0001$ & $2.50(1.20-7.40)$ \\
\hline CA19-9, U/mL & $11(6.8-116.9)$ & $7.75(4.15-14.2)$ & $<0.0001$ & $9.00(5.0-20.30)$ \\
\hline Body mass index & $26.27(24.2-29.74)$ & $26.59(24.54-29.4)$ & 0.749 & $26.45(24.22-29.40)$ \\
\hline \multicolumn{5}{|l|}{ Stage } \\
\hline 1 & $1(1.0 \%)$ & $8(6.3 \%)$ & \multirow[t]{4}{*}{$<0.0001$} & $9(3.9 \%)$ \\
\hline 2 & $29(27.6 \%)$ & $72(56.7 \%)$ & & $101(43.5 \%)$ \\
\hline 3 & $32(30.5 \%)$ & $41(32.3 \%)$ & & $73(31.5 \%)$ \\
\hline 4 & $43(41 \%)$ & $6(4.7 \%)$ & & $49(21.1 \%)$ \\
\hline
\end{tabular}

Abbreviations: ADAMTS13, a disintegrin and metalloproteinase with thrombospondin type-I repeats 13; VWF, von Willebrand factor.

${ }^{a}$ Nonfatal thromboembolic event and/or death of any cause.

${ }^{b}$ Median and interquartile range or number and percentages.

${ }^{c}$ Chi-square test or Mann-Whitney t-test.

all-cause mortality in this study. In the follow-up period, 97 patients died and/or 26 had thrombotic complication.

\section{Laboratory Measurements}

ADAMTS13 enzyme activity was determined in citrated plasma samples using the fluorogenic substrate FRETSVWF73 as previously described. ${ }^{24}$

VWF:Ag level was measured in EDTA plasma by ELISA as described. ${ }^{25}$ Primary antibodies and horseradish peroxidiselabeled polyclonal rabbit anti-human VWF antibodies were used for the detection (both from Dako, Glostrup, Denmark). ${ }^{24}$ Determination of the additional laboratory parameters including complete blood counts, clinical chemistry and tumour markers were completed by Roche Integra 800 analyser, and a Cell-Dyn 3500 haematology analyser.

VWF multimer analysis was performed with patients' plasma samples which were normalized to VWF:Ag concentration based on VWF:Ag ELISA results. VWF multimer gels $(1.2 \%(\mathrm{w} / \mathrm{v})$ type VIIA agarose [Sigma-Aldrich] running gel and SeaKem HGT(P) agarose [Lonza] stacking gel) were loaded with denaturated normalized plasmas diluted in sample buffer. Gels were run overnight and blotted into a nitrocellulose membrane (Whatman Protran). Membranes were blocked in $1.5 \%$ milk shaking and probed with rabbit polyclonal antihuman VWF (DAKO) at 1:2.000, conjugated with goat anti-rabbit HRP antibody (Biorad) and developed using chemiluminescent substrate (Sigma-Aldrich). Digital images were acquired, densitometry waveforms were generated using Alpha Innotech (Fluorchem Ltd) and multimer structures of patients were compared with normal pooled plasma (NPP). A sample was defined as positive for the presence of ultra-large von Wil- lebrand factor (ULVWF) if bands with more than 15 multimers were present.

\section{Statistical Analysis}

The continuous variables reported in this study showed skewed distribution according to the results of the ShapiroWilk test deviated from the normal distribution. Therefore, for descriptive purposes, the values of each measurement are given as median and 25th to 75th percentile or as numbers (percent) and non-parametric tests (Spearman's rank correlation or Mann-Whitney U-test) were used for group comparisons. For categorical variables, Pearson's chi-square test was applied. The correlation between the different variables was determined by using Spearman's correlation analysis.

Multivariable Cox proportional hazard models were fitted to assess the effect of platelet count, VWF:Ag level and ADAMTS13 activity on the event-free survival in patients. Survival times were measured from the diagnosis of colorectal cancer and patients were censored at the time of death, at the time of nonfatal thromboembolic event, or at the end of the follow-up period if still alive (month 60). Potential predictors of events and confounders were entered into the models as categorical variables, and high or low predictor groups were formed based on the results of receiver operating characteristic (ROC) curve analysis. All of the survival models were adjusted to age, gender and stage of disease. The proportional hazards assumption was confirmed graphically by Kaplan-Meier analysis, survival curves were compared with log-rank (Mantel-Cox) test. The results of the Cox regression models are presented as hazard ratios (HRs) with corresponding 95\% confidence intervals (CIs). With a sample size of 232 and a predicted event rate around 
0.5, the precision of Cox regression models are considered satisfactory, as reflected by the sufficiently narrow $\mathrm{CI}$ ranges. The presence of collinearity in the multivariable models was inferred by calculating variance inflation factors (VIF). IBM SPSS Statistics 20 and Graph Pad Prism 5 software were used for the statistical analysis and presentation of data. Two-tailed $p$-values were calculated and significance level of $p<0.05$ was used, if not otherwise stated.

\section{Results}

A total of 232 patients with colorectal cancer, with a mean age of 68.5 years and slight male predominance (-Table $\mathbf{1}$ ), were enrolled in the study and followed up for 5 years. Over the 5-year-long follow-up period, 96 patients died and/or 26 had a thromboembolic event. Clinical and laboratory variables of the cohort are summarized in - Table 1. Among these 26 patients, there were 8 patients who had only thromboembolic complications but did not die within the follow-up period, whereas 2 patients had a fatal thromboembolic event, and 18 patients died within the follow-up period after surviving thromboembolic event. Accordingly, during follow-up, 105 patients suffered nonfatal thromboembolic events, or died.

As presented in - Table 1, mortality and/or thromboembolic events were associated with significantly higher platelet count $(p=0.013)$, VWF:Ag level $(p=0.001)$, carcinoembryonic antigen- (CEA) and CA19-9 (cancer antigen 19-9) levels (all $p<0.0001)$, and lower ADAMTS13 activity $(p<0.0001)$. Similarly, advanced disease stage was also associated with poor outcome ( $p<0.0001$ ). In 26 cases, thromboembolic complication occurred during the follow-up period. We found that patients with thromboembolism had lower ADAMTS13 activity (63.23\%; 55.14-68.97\%; $p<0.0001$ ) and higher VWF:Ag (665.74 ng/mL; 387.56-2,092.92 ng/mL; $p=0.048$ ) levels, when compared with patients without events (ADAMTS13 activity: 80.27\% [67.58-98.92\%]; VWF:Ag level: $514.43 \mathrm{ng} / \mathrm{mL}$ [280.73-964.27 ng/mL]), which also supports the hypothesis that the observed decrease of ADAMTS13 activity in colorectal cancer patients is clinically relevant. There was no correlation between VWF:Ag levels, ADAMTS13 activity and platelet count using Spearman's correlation (ADAMTS13-VWF:Ag: $r=-0.114, p=0.08$; ADAMTS13-platelet count: $r=0.043$, $p=0.526$; VWF:Ag-platelet count: $r=0.022, p=0.746$ ).

Increased VWF:Ag levels and decreased ADAMTS13 activity were observed for many of our patients. There were 49 patients with high VWF:Ag level and low ADAMTS13 activity which is $21.6 \%$ of our patients (the other groups were as follows: low ADAMTS13-low VWF:AG: $n=16$ [7\%]; high ADAMTS13 activity-low VWF:Ag: $n=46$ [20.3\%]; high ADAMTS13 activityhigh VWF:AG: $n=116$ [51.1\%]). Decreased ADAMTS13 activity with the presence of ultra-large VWF multimers was observed in several diseases such as TTP, HUS, preeclampsia, liver cirrhosis, chronic renal insufficiency and acute inflammatory states, ${ }^{26}$ and was found to be linked to higher risk of thromboembolism. ${ }^{27}$ Therefore, by analysing the presence of ultra-large VWF (ULVWF) oligomers in a selected subset of patients (in 55 plasma samples obtained from patients in the highest VWF:Ag level group associated with the lowest

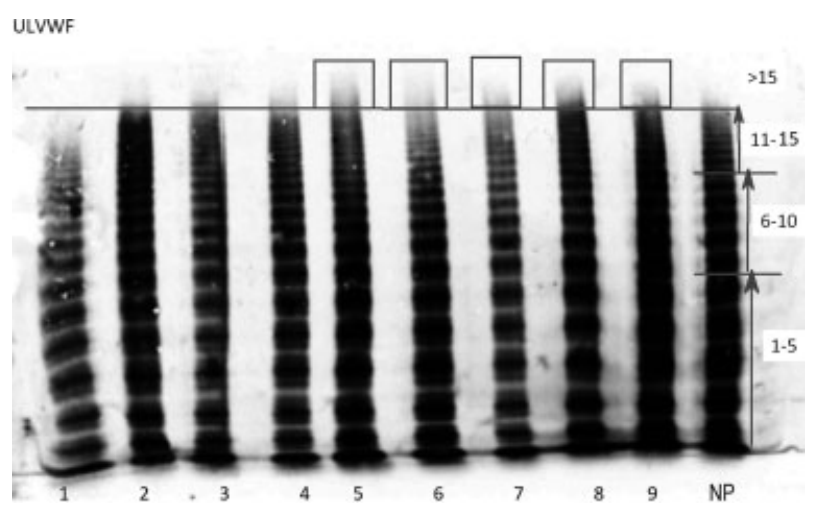

Fig. 1 Multimeric analysis of plasma von Willebrand factor (VWF). Oligomeric structure of VWF was determined by agarose electrophoresis, ULVWF was defined if bands representing more than 15-mer oligomers were present in patients' plasma samples. Oligomeric structures of nine representative samples are shown, samples 1 to 4 are negative, whereas samples 5 to 9 are positive for ULVWF (marked by squares). Normal human plasma (NP) is presented as negative control.

ADAMTS13 activity [ $n=28]$ or vice versa [ $n=27]$ ), we further examined if the observed increase of VWF:Ag with the decrease of ADAMTS13 activity is clinically relevant or not. Oligomeric structure of VWF was determined by agarose electrophoresis, and a sample was considered positive for ULVWF if VWF with more than 15 multimers was present (-Fig. 1). A significantly ( $p=0.01$ ) increased frequency (67\%) of ULVWF was observed in the high VWF:Ag and low ADAMTS13 activity group of colorectal cancer patients, when compared with the low VWF:Ag and high ADAMTS13 activity group (32\%, - Supplementary Table S1 [online only]), indicating clinical relevance of this observation. Taken together, these data support that lower ADAMTS13 activity and higher VWF:Ag level could be useful information according to the prothrombotic state in the patients.

Next, we examined whether baseline platelet count, VWFantigen level and ADAMTS13 activity are associated with disease stage (-Fig. 2). We merged stages 1 and 2 because of the small number of cases. Significantly higher platelet counts $(p=0.005)$, VWF:Ag levels $(p=0.008)$ and decreased ADAMTS13 activity ( $p=0.006)$ were observed in the group of patients with metastatic disease (stage 4) when compared with patients in stages 1 and 2 or when compared the patients with lymph node metastasis (stage 3 , all $p<0.05$, except for VWF:Ag where this difference was nonsignificant). We also analysed the association between platelet counts, ADAMTS13 activity and VWF:Ag levels in the total cohort and no correlation was observed between any of the markers.

To examine in multivariable models whether platelet count, VWF:Ag level or ADAMTS13 activity could influence or have any relation with event-free survival, patients were categorized as those with "high" or "low" marker levels. The cut-off values (indicated in - Tables 2-4), with optimum sensitivity and specificity for all-cause mortality of the above-mentioned variables were defined by ROC curve analysis. The distribution of the clinical and laboratory characteristics of patients with high or low platelet count, VWF:Ag level and ADAMTS13 activity is shown in - Tables 2 to 4 . 

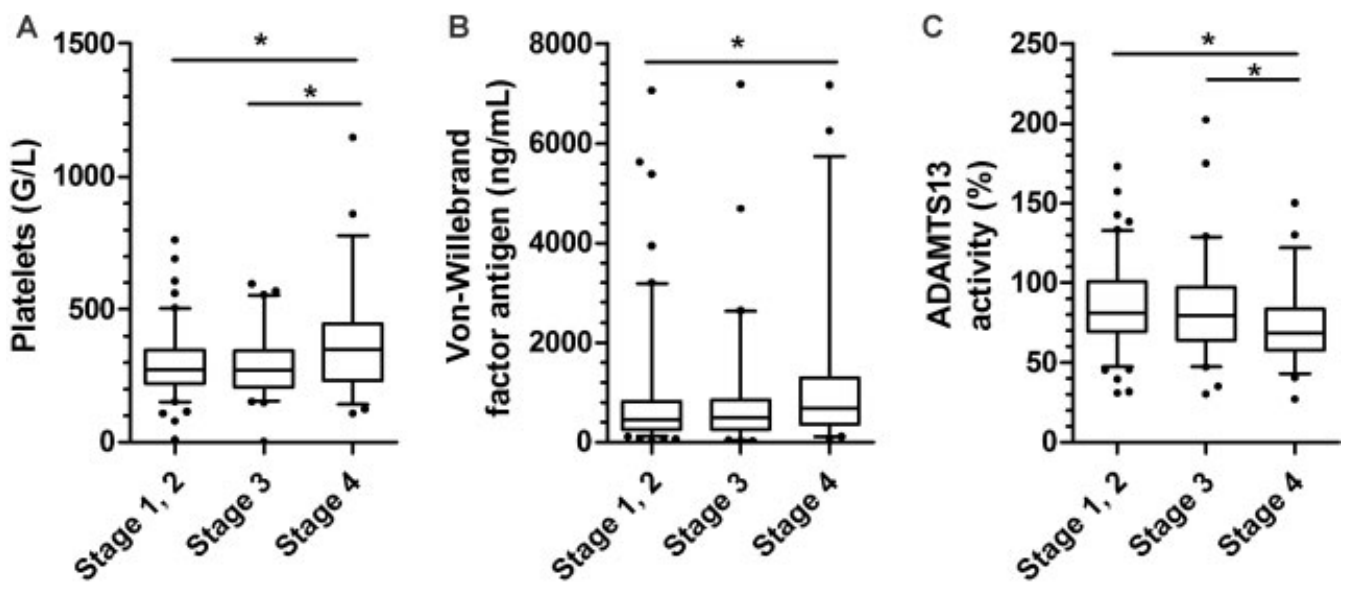

Fig. 2 Box plot of the studied three variables according to different tumour stages. (Panel A) Platelet count; (Panel B) von Willebrand factor antigen level; (Panel C): ADAMTS13 activity. The difference between stages 1,2 and 4 is significant $(p<0.05)$ in all panels. In Panels A and C, there is a significant difference between Stages 3 and 4 as well (both $p<0.05$ ). Horizontal lines show medians. The boxes enclose interquartile range and whiskers indicate the 5 th to 95 th percentiles of the groups. ${ }^{*} p<0.05$.

In addition, we examined the levels of different tumour markers in these groups (- Tables 2-4). Higher CA19-9 levels were associated with elevated levels of VWF:Ag and decreased ADAMTS13 activity, whereas increased platelet count was associated with higher CEA levels ( - Table 2).

By examining the association between TNM classification and the above markers, we found that advanced stages were significantly associated with high platelet $\operatorname{count}(p=0.001)$ and lower ADAMTS13 activity $(p=0.011)$, and a borderline association $(p=0.074)$ with high VWF:Ag level was also observed.

The cumulative event-free survival of patients with different platelet counts, ADAMTS13 activity and VWF:Ag levels is displayed in -Fig. 3. Results of the Kaplan-Meier analysis showed that lower platelet counts $(p<0.0001)$, lower
VWF:Ag $(p=0.0008)$ levels, and higher ADAMTS13 activity $(p<0.0001)$ were associated with better event-free survival. Overall event-free survival at the end of follow-up was $61.68 \%$ in the low and $38.57 \%$ in the high platelet count group, $32.8 \%$ in the low and $64.02 \%$ in the high ADAMTS13 activity group and $62.5 \%$ in the low and $49 \%$ in the high VWF:Ag group.

Finally, to investigate the association between overall eventfree survival and the three studied variables, multivariate Cox proportional hazard models were generated, and the results are shown in - Table 5 . The baseline model (Model 1) included age, gender and disease stage, and all subsequent models were adjusted for these three variables. Next, platelet count, ADAMTS13 activity and VWF:Ag level (as binary variables) were incorporated (Models 2, 3 and 4) and all of these variables

Table 2 Clinical and laboratory characteristics of patients with high or low platelet count

\begin{tabular}{|c|c|c|c|}
\hline & Platelet count $\leq 348.5 \mathrm{G} / \mathrm{L}^{\mathrm{b}}$ & Platelet count $>348.5 \mathrm{G} / \mathrm{L}$ & $p$-Value ${ }^{c}$ \\
\hline Number of patients & 154 & 70 & - \\
\hline No event/event ${ }^{\mathrm{a}}$ & $95 / 59$ & $27 / 43$ & 0.001 \\
\hline Males/Females & $103 / 51$ & $30 / 40$ & 0.001 \\
\hline Age at diagnosis, y & $68.35(59.60-73.30)$ & $68.70(60.40-74.90)$ & 0.407 \\
\hline ADAMTS13 activity, $\%$ & $79.31(65.04-98.69)$ & $75.89(65.8-94.18)$ & 0.473 \\
\hline VWF antigen, $\mathrm{ng} / \mathrm{mL}$ & $501.28(262.72-1,048.03)$ & $576.60(348.14-1011.30)$ & 0.405 \\
\hline Carcinoembryonic antigen, $\mu \mathrm{g} / \mathrm{L}$ & $2.00(1.10-3.50)$ & $5.80(2.20-19.70)$ & $<0.0001$ \\
\hline CA19-9, U/mL & $9.10(5.30-16.70)$ & $8.30(4.20-63.50)$ & 0.754 \\
\hline Body mass index & $27.17(24.84-29.74)$ & $26.11(22.31-29.75)$ & 0.111 \\
\hline \multicolumn{4}{|l|}{ Stage } \\
\hline 1 & $9(5.8 \%)$ & $0(0.0 \%)$ & \multirow[t]{4}{*}{0.001} \\
\hline 2 & $73(47.4 \%)$ & $26(37.1 \%)$ & \\
\hline 3 & $50(32.5 \%)$ & 19 (27.1\%) & \\
\hline 4 & $22(14.3 \%)$ & $25(35.7 \%)$ & \\
\hline
\end{tabular}

Abbreviations: ADAMTS13, a disintegrin and metalloproteinase with thrombospondin type-I repeats 13; VWF, von Willebrand factor.

${ }^{a}$ Nonfatal thromboembolic event and/or death of any cause.

${ }^{\mathrm{b}}$ Median and interquartile range or number and percentages.

${ }^{c}$ Chi-square test or Mann-Whitney $t$-test. 
Table 3 Clinical and laboratory characteristics of patients with high or low ADAMTS13 activity

\begin{tabular}{|c|c|c|c|}
\hline & ADAMTS13 activity $\leq 66.53 \%^{\mathrm{b}}$ & ADAMTS13 activity $>66.53 \%$ & $p$-Value ${ }^{c}$ \\
\hline Number of patients & 67 & 164 & - \\
\hline No event/event ${ }^{\mathrm{a}}$ & $22 / 45$ & $105 / 59$ & $<0.0001$ \\
\hline Males/Females & $47 / 20$ & $90 / 74$ & 0.032 \\
\hline Age at diagnosis, $y$ & $72.40(67.80-78.60)$ & $65.45(58.10-71.80)$ & $<0.0001$ \\
\hline Platelet G/L & $266(214-353)$ & $291(229-367)$ & 0.343 \\
\hline VWF antigen, ng/mL & $635.40(318.0-1,286.51)$ & $502.62(280.73-904.66)$ & 0.207 \\
\hline Carcinoembryonic antigen, $\mu \mathrm{g} / \mathrm{L}$ & $3.20(1.10-28.90)$ & $2.30(1.20-5.20)$ & 0.090 \\
\hline CA19-9, U/mL & $12.40(7.10-49.40)$ & $8.10(4.50-16.70)$ & 0.008 \\
\hline Body mass index & $26.11(24.78-29.08)$ & $27.04(23.67-29.40)$ & 0.734 \\
\hline \multicolumn{4}{|l|}{ Stage } \\
\hline 1 & $2(3.0 \%)$ & $7(4.3 \%)$ & \multirow[t]{4}{*}{0.011} \\
\hline 2 & $20(29.9 \%)$ & $81(49.4 \%)$ & \\
\hline 3 & $23(34.3 \%)$ & $50(30.5 \%)$ & \\
\hline 4 & $22(32.8 \%)$ & $26(15.9 \%)$ & \\
\hline
\end{tabular}

Abbreviations: ADAMTS13, a disintegrin and metalloproteinase with thrombospondin type-I repeats 13; VWF, von Willebrand factor.

${ }^{a}$ Nonfatal thromboembolic event and/or death of any cause.

${ }^{\mathrm{b}}$ Median and interquartile range or number and percentages.

${ }^{\mathrm{C}} \mathrm{Chi}$-square test or Mann-Whitney $t$-test.

Table 4 Clinical and laboratory characteristics of patients with high or low von Willebrand factor antigen

\begin{tabular}{|c|c|c|c|}
\hline & $\begin{array}{l}\text { von Willebrand factor } \\
\text { antigen } \leq 305.6 \mathrm{ng} / \mathrm{mL}^{\mathrm{b}}\end{array}$ & $\begin{array}{l}\text { von Willebrand factor } \\
\text { antigen }>305.6 \mathrm{ng} / \mathrm{mL}\end{array}$ & $p$-Value ${ }^{c}$ \\
\hline Number of patients & 62 & 165 & - \\
\hline No event/event ${ }^{\mathrm{a}}$ & $45 / 17$ & $81 / 84$ & 0.002 \\
\hline Males/Females & $40 / 22$ & $94 / 71$ & 0.303 \\
\hline Age at diagnosis, $y$ & $65.55(58.50-73.00)$ & $68.80(60.60-74.70)$ & 0.067 \\
\hline Platelet G/L & $282(223-339.5)$ & $291(227-368)$ & 0.298 \\
\hline ADAMTS13 activity, \% & $83.88(66.42-98.45)$ & $77.74(65.02-95.12)$ & 0.377 \\
\hline Carcinoembryonic antigen, $\mu \mathrm{g} / \mathrm{L}$ & $2.15(1.10-4.20)$ & $2.70(1.20-8.50)$ & 0.134 \\
\hline CA19-9, U/mL & $7.45(4.20-14.20)$ & $9.50(5.60-21.90)$ & 0.03 \\
\hline Body mass index & $26.20(22.59-28.23)$ & $26.93(24.39-29.76)$ & 0.164 \\
\hline \multicolumn{4}{|l|}{ Stage } \\
\hline 1 & $2(3.2 \%)$ & $7(4.2 \%)$ & \multirow[t]{4}{*}{0.074} \\
\hline 2 & $33(53.2 \%)$ & $67(40.6 \%)$ & \\
\hline 3 & $21(33.9 \%$ & $50(30.3 \%)$ & \\
\hline 4 & $6(9.7 \%)$ & $41(24.8 \%)$ & \\
\hline
\end{tabular}

Abbreviation: ADAMTS13, a disintegrin and metalloproteinase with thrombospondin type-I repeats 13 .

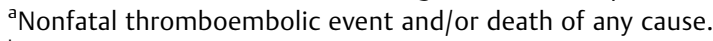

${ }^{\mathrm{b}}$ Median and interquartile range or number and percentages.

${ }^{\mathrm{c} C h i}$-square test or Mann-Whitney t-test.

turned out to be age-, gender- and stage-independent predictors of mortality or thromboembolic complications (all HR $>1.7$, $p<0.05$, - Supplementary Table $\mathbf{5 2}$ [online only]). When we further adjusted Models 2 to 4 also for the well-known tumour markers of colorectal cancer (CEA, CA19-9), which were associated with mortality or thromboembolic complications
(-Table 1), all of the examined variables remained significant predictors of event-free state except ADAMTS13 activity (platelet count in Model 1 adjusted for CEA: HR 1.908, CI 1.214-3; ADAMTS13 activity in Model 2 adjusted for CA19-9: HR 1.519, CI 0.955-2.416; VWF:Ag level in Model 3 adjusted for CA19-9: HR 2.021; CI 1.169-3.494). We also examined the relationship of 

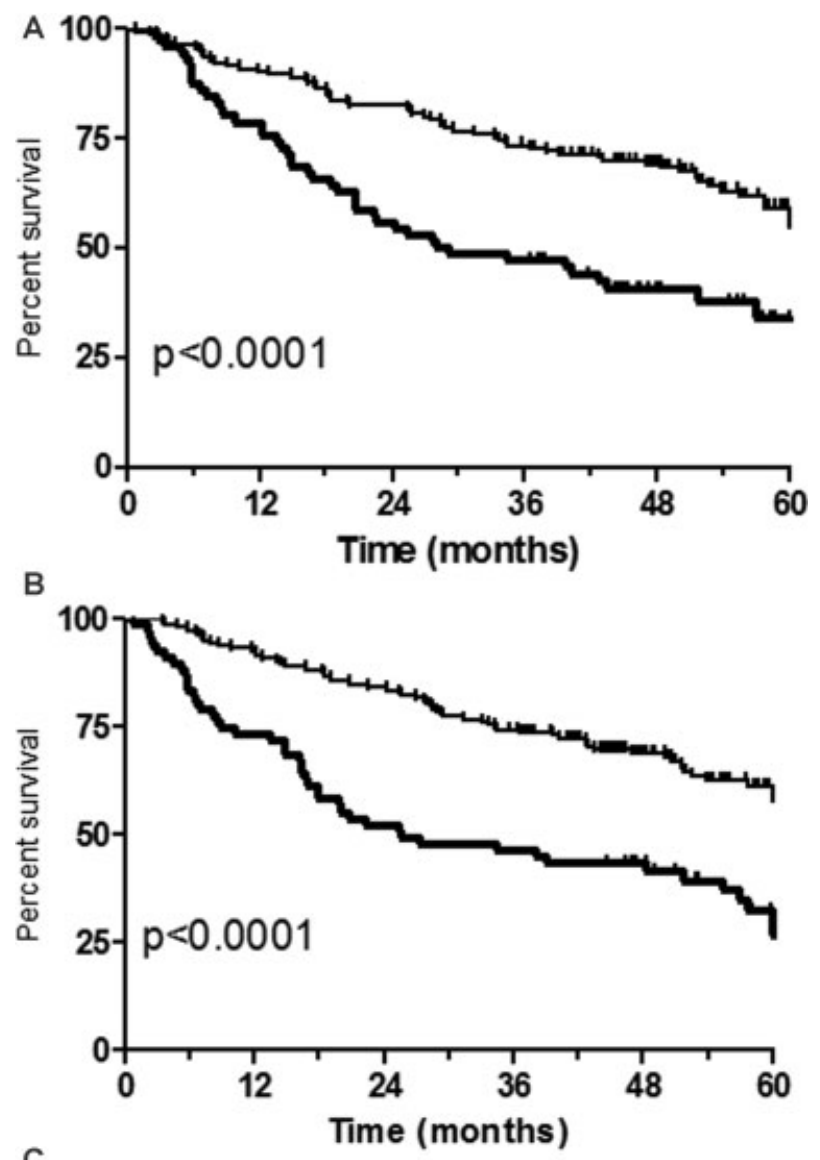

Table 5 Model 5: Relationship between platelet count, ADAMTS13 activity and von Willebrand factor antigen and event-free survival of 232 patients with colorectal cancer during the 5-year-long follow-up period

\begin{tabular}{|c|c|c|c|}
\hline & HR & $\mathrm{Cl}$ & $p$-Value \\
\hline $\begin{array}{l}\text { Age } \\
\text { (by } 10 \text { y of increase) }\end{array}$ & 1.277 & $1.034-1.576$ & 0.023 \\
\hline \multicolumn{4}{|l|}{ Gender } \\
\hline $\begin{array}{l}\text { Female } \\
\text { Male }\end{array}$ & $\begin{array}{l}1 \\
0.876\end{array}$ & $0.562-1.366$ & 0.559 \\
\hline \multicolumn{4}{|l|}{ Stage } \\
\hline $\begin{array}{l}1,2,3 \\
4\end{array}$ & $\begin{array}{l}1 \\
3.638\end{array}$ & $2.345-5.645$ & $<0.0001$ \\
\hline \multicolumn{4}{|l|}{ Platelet count } \\
\hline $\begin{array}{l}\leq 348.5 \mathrm{G} / \mathrm{L} \\
>348.5 \mathrm{G} / \mathrm{L}\end{array}$ & $\begin{array}{l}1 \\
1.967\end{array}$ & $1.257-3.077$ & 0.003 \\
\hline \multicolumn{4}{|l|}{ ADAMTS13 activity } \\
\hline $\begin{array}{l}>66.53 \% \\
\leq 66.53 \%\end{array}$ & $\begin{array}{l}1 \\
1.903\end{array}$ & $1.22-3.577$ & 0.003 \\
\hline \multicolumn{4}{|c|}{ von Willebrand factor antigen } \\
\hline $\begin{array}{l}\leq 305.6 \mathrm{ng} / \mathrm{mL} \\
>305.6 \mathrm{ng} / \mathrm{mL}\end{array}$ & $\begin{array}{l}1 \\
2.089\end{array}$ & $1.169-3.651$ & 0.007 \\
\hline
\end{tabular}

Abbreviation: ADAMTS13, a disintegrin and metalloproteinase with thrombospondin type-I repeats 13 .

Note: The effect of different other demographic and clinical variables was calculated by multivariable Cox regression analysis.

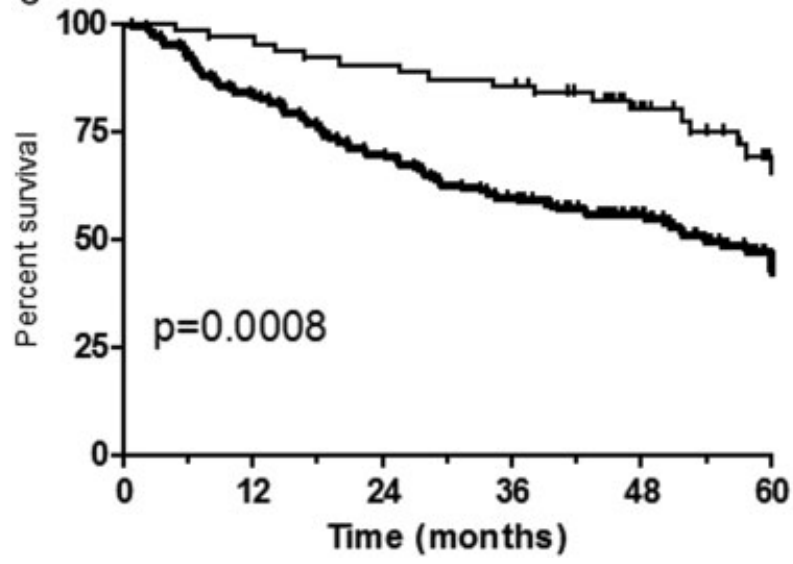

Fig. 3 Kaplan-Meier analysis of patient groups with higher and lower levels of the studied variables. (Panel A) Platelet count, thick line: $>348.5 \mathrm{G} / \mathrm{L}$, thin line: $\leq 348.5 \mathrm{G} / \mathrm{L}$; (Panel B): von Willebrand factor antigen level, thick line: $>305.6 \mathrm{ng} / \mathrm{mL}$, thin line: $\leq 305.6 \mathrm{ng} / \mathrm{mL}$; (Panel C): ADAMTS13 activity, thick curve: $\leq 66.53 \%$, thin curve: $>66.53 \%$. $p$-Value of log-rank test is indicated.

these variables for an aggregate effect on event-free survival (Model 5, - Table 5) and observed that platelet count (HR: 1.967, CI: 1.257-3.077), ADAMTS13 activity (HR: 1.903, CI: 1.22-3.577) and VWF:Ag level (HR: 2.089, CI: 1.169-3.651) remained significant predictors of mortality or thromboembolic complications in the final multivariable model. Multiple collinearity was not present in the final multivariable model, since all VIF scores were in the range 1 to 10 .

Based on all of the above observations, careful analysis and interpretation of platelet count, ADAMTS13 activity and VWF:Ag level may yield clinically important information in colorectal cancer, since these markers are associated with increased prothrombotic risk and independently predict worse outcome.

\section{Discussion}

In this cohort study with a 5-year follow-up, we report that platelet count, ADAMTS13 activity and VWF:Ag levels are related to different stages of colorectal cancer and have prognostic significance. Higher platelet count, VWF:Ag level and lower ADAMTS13 activity are associated with advanced stage and independently and significantly predicts poor overall event-free survival. The novelty of this study is the demonstration of a clinically relevant decrease of ADAMTS13 activity with increased thromboembolic risk in colorectal cancer, and the independent association of higher platelet count, higher VWF: Ag levels and lower ADAMTS13 activity with poor thromboembolic event-free survival in colorectal cancer patients. Higher occurrence of ultra-large VWF oligomers in colorectal cancer patients with increased VWF:Ag and decreased ADAMTS13 activity strongly supported clinical relevance of this observation. Taken together, previous literature results ${ }^{20,21}$ and our observations in colorectal cancer patients are convincingly supporting the link between increased VWF:Ag, decreased ADAMTS13 activity and increased prothrombotic risk. 
An important observation of the current study is the demonstration of association between thrombocytosis with poor 5-year event-free survival in colorectal cancer, confirming previous results. ${ }^{6-13,15}$ Elevated platelet count was associated with metastatic (stage 4) disease, whereas patients with locoregional disease (stage 3 ) had similar platelet counts as patients with stage 1 to 2 diseases (-Fig. 1). The association between metastatic disease and thrombocytosis is intriguing. During metastasis formation, cancer cells escape from the primary tumour and get to distal sites while the cells are hidden from the tumour surveillance mechanisms. ${ }^{28}$ There is growing evidence of an interaction between tumour cells and platelets which may promote tumour cell survival and tumour progression. The surface receptors of platelets (adhesion receptors such as GP IIb/IIIa, GP Ib-IX-V and integrins $\alpha v \beta 3$ ) can directly interact with tumour cells leading to the formation of heteroaggregates. ${ }^{5}$ The interaction of platelets with tumour cells may also lead to platelet activation, aggregation ${ }^{5}$ and degranulation, 28 a process called tumour cell-induced platelet aggregation (TCIPA). ${ }^{4,28}$ Factors released from the $\alpha$-granules include PDGF, VEGF, IGF-1 and TGFß, which may contribute to tumour growth, spread and neovascularization.

Both the thrombin- and the VEGF-dependent pathways induce endothelial cell activation followed by Weibel-Palade body exocytosis and the release of $\mathrm{VWF}^{29}$ Von Willebrand factor is a key regulator of primary haemostasis, ${ }^{16}$ as the released multimer can bind to platelet surface receptors and promote the formation of the platelet thrombus. ${ }^{17,30}$ Recent observations obtained from human clinical samples support a VWF-mediated mechanism in tumour progression and angiogenesis, where tumour-derived VEGF plays a pivotal role. Analysis of human blood samples and tumour tissue revealed that VWF release combined with a local inhibition of proteolytic activity and protein expression of ADAMTS13 accounted for a tumour propagating procoagulatory milieu. ${ }^{31}$ Increased plasma levels of VWF:Ag were associated with poor prognosis in different types of cancer (ovarian, bladder, gastric and colon). ${ }^{16,17,32,33}$ Increased expression of VWF was observed in the tumour compared with the non-tumoural parenchyma in gastric cancer, and late gastric cancer showed significantly higher levels of VWF:Ag. ${ }^{16}$ The GPIb $\alpha$ receptor can be expressed by various tumour cells, such as human breast cancer cells, and it was suggested that the function of this receptor on tumour cells differs in its adhesive interaction with VWF from that of platelets. ${ }^{34,35}$ The most active, ultra-large form of VWF is released from the endothelial Weibel-Palade bodies, ${ }^{36}$ and ADAMTS13-mediated proteolytic processes may be decreased. Hence, the cumulative action of these processes may lead to the formation of firm tumour-platelet aggregates linked by fibres of ultra-large VWF. ${ }^{31}$ Our observations are in line with this mechanism, since we were able to show in this study the independent association of decreased ADAMTS13 activity (72.28\% on average) and increased VWF:Ag levels (677.86 $\mathrm{ng} / \mathrm{mL}$ on average) with poor event-free survival of patients with colorectal cancer. The analysis of the oligomeric form of VWF showed that ultra-large oligomers occurred more frequently in patients with high VWF:Ag levels and low ADAMTS13 activity, when compared with those with low VWF:Ag levels and high ADAMTS13 activity group (-Supplementary Table S1 [online only]). Decreased ADAMTS13 activity with increased VWF:Ag levels was associated with increased occurrence of thromboembolic events which support the clinical relevance of this observation (-Supplementary Table S1 [online only]). Decreased activity of ADAMTS13 was observed in clinical studies in the plasma of patients with different malignancies mostly in advanced, disseminated stages; ${ }^{19,22}$ however, this is the first study providing epidemiologic evidence for the association between decreased ADAMTS13 activity and poor event-free survival in a 5-year-long follow-up study. Our current observations are in line with results of previous studies and may support the important role of ADAMTS13 and VWF in the pathomechanism of cancer-related thromboembolism and mortality.

In summary, this is the first observational study reporting platelet count, ADAMTS13 activity and VWF:Ag level simultaneously in colorectal cancer. We showed that thrombocytosis, elevated VWF:Ag levels and decreased VWF-processing protease activity independently associate with poor event-free survival. Therefore, these epidemiological data may support prior observations obtained from animal clinical studies on the contribution of tumour cell-platelet heteroaggregates, held together by VWF-fibres, in the propagation of tumour progression. These observations may therefore provide further support to plan novel therapies targeting endothelial cell activation or microthrombi formation in cancer treatment.

\section{What is known about this topic?}

- Metastasis formation is the leading cause of cancerrelated death. There is growing evidence of an interaction between tumour cells and platelets which may influence tumour progression and metastasis formation and may be regulated by the VWF.

- Preoperative thrombocytosis and increased VWF:Ag level are associated with poor prognosis in different types of cancer. In disseminated malignancies, decreased ADAMTS13 serum levels were measured.

- It is important to find new reliable markers which can predict prognosis in colorectal cancer.

\section{What does this paper add?}

- We measured ADAMTS13 activity in a large cohort of colorectal cancer patients at the first time and observed the association of decreased activity-together with increased platelet count and VWF:Ag level-with poor survival without thrombotic complications.

- We report that increased platelet count, VWF:Ag level and decreased ADAMTS13 activity are all independently associated with advanced stage and poorer event-free survival.

- These observational data may fuel further research on tumour cell-platelet heteroaggregates, to explore the role of VWF-fibres in tumour progression and metastasis formation and may finally highlight novel targets of therapy. 


\section{Conflicts of interest}

None declared.

\section{Acknowledgements}

We thank I. Szigeti, Zs. Szendrei, M. Kókai, E. Kertész and J. Varga for providing technical assistance with sample collection. We also thank Á. Holczbauer, E. Kajdácsi, B. Mikes and L. Laszip for their supporting work in this study. This work was supported by the Hungarian Academy of Sciences (research grant TKI2017-02064).

\section{References}

1 Vogelaar I, van Ballegooijen M, Schrag D, et al. How much can current interventions reduce colorectal cancer mortality in the U.S.? Mortality projections for scenarios of risk-factor modification, screening, and treatment. Cancer 2006;107(07):1624-1633

2 Hur K. MicroRNAs: promising biomarkers for diagnosis and therapeutic targets in human colorectal cancer metastasis. BMB Rep 2015;48(04):217-222

3 Siegel R, Desantis C, Jemal A. Colorectal cancer statistics, 2014. CA Cancer J Clin 2014;64(02):104-117

4 Goubran HA, Stakiw J, Radosevic M, Burnouf T. Platelet-cancer interactions. Semin Thromb Hemost 2014;40(03):296-305

5 Tesfamariam B. Involvement of platelets in tumor cell metastasis. Pharmacol Ther 2016;157:112-119

6 Allensworth SK, Langstraat CL, Martin JR, et al. Evaluating the prognostic significance of preoperative thrombocytosis in epithelial ovarian cancer. Gynecol Oncol 2013;130(03):499-504

$7 \mathrm{Kim} \mathrm{KH}$, Park TY, Lee JY, et al. Prognostic significance of initial platelet counts and fibrinogen level in advanced non-small cell lung cancer. J Korean Med Sci 2014;29(04):507-511

$8 \mathrm{Kim} \mathrm{M}$, Chang H, Yang HC, et al. Preoperative thrombocytosis is a significant unfavorable prognostic factor for patients with resectable non-small cell lung cancer. World J Surg Oncol 2014; $12: 37$

9 Nozoe T, Iguchi T, Maeda T, Kohno M, Ezaki T. Simple criteria to predict prognosis in colorectal carcinoma based on lymphopenia and thrombocytosis. Mol Clin Oncol 2013;1(01):171-174

10 Sasaki K, Kawai K, Tsuno NH, Sunami E, Kitayama J. Impact of preoperative thrombocytosis on the survival of patients with primary colorectal cancer. World J Surg 2012;36(01):192-200

11 Ishizuka M, Nagata H, Takagi K, Iwasaki Y, Kubota K. Preoperative thrombocytosis is associated with survival after surgery for colorectal cancer. J Surg Oncol 2012;106(07):887-891

12 Monreal M, Fernandez-Llamazares J, Piñol M, et al. Platelet count and survival in patients with colorectal cancer-a preliminary study. Thromb Haemost 1998;79(05):916-918

13 Kawai K, Kitayama J, Tsuno NH, Sunami E, Watanabe T. Thrombocytosis before pre-operative chemoradiotherapy predicts poor response and shorter local recurrence-free survival in rectal cancer. Int J Colorectal Dis 2013;28(04):527-535

14 Wan S, Lai Y, Myers RE, et al. Preoperative platelet count associates with survival and distant metastasis in surgically resected colorectal cancer patients. J Gastrointest Cancer 2013;44(03): 293-304

15 Stone RL, Nick AM, McNeish IA, et al. Paraneoplastic thrombocytosis in ovarian cancer. N Engl J Med 2012;366(07):610-618

16 Yang X, Sun HJ, Li ZR, et al. Gastric cancer-associated enhancement of von Willebrand factor is regulated by vascular endothelial growth factor and related to disease severity. BMC Cancer 2015;15:80

17 Luo GP, Ni B, Yang X, Wu YZ. von Willebrand factor: more than a regulator of hemostasis and thrombosis. Acta Haematol 2012;128 (03):158-169

18 Lippok S, Radtke M, Obser T, et al. Shear-induced unfolding and enzymatic cleavage of full-length VWF multimers. Biophys J 2016;110(03):545-554

19 Mannucci PM, Karimi M, Mosalaei A, Canciani MT, Peyvandi F. Patients with localized and disseminated tumors have reduced but measurable levels of ADAMTS-13 (von Willebrand factor cleaving protease). Haematologica 2003;88(04):454-458

20 Francis KK, Kalyanam N, Terrell DR, Vesely SK, George JN. Disseminated malignancy misdiagnosed as thrombotic thrombocytopenic purpura: A report of 10 patients and a systematic review of published cases. Oncologist 2007;12(01):11-19

21 Lechner K, Obermeier HL. Cancer-related microangiopathic hemolytic anemia: clinical and laboratory features in 168 reported cases. Medicine (Baltimore) 2012;91(04):195-205

22 Oleksowicz L, Bhagwati N, DeLeon-Fernandez M. Deficient activity of von Willebrand's factor-cleaving protease in patients with disseminated malignancies. Cancer Res 1999;59(09):2244-2250

23 Elyamany G, Alzahrani AM, Bukhary E. Cancer-associated thrombosis: an overview. Clin Med Insights Oncol 2014;8:129-137

24 Gombos T, Makó V, Cervenak L, et al. Levels of von Willebrand factor antigen and von Willebrand factor cleaving protease (ADAMTS13) activity predict clinical events in chronic heart failure. Thromb Haemost 2009;102(03):573-580

25 Cejka J. Enzyme immunoassay for factor VIII-related antigen. Clin Chem 1982;28(06):1356-1358

26 Pimanda J, Hogg P. Control of von Willebrand factor multimer size and implications for disease. Blood Rev 2002;16(03):185-192

27 Arya M, Anvari B, Romo GM, et al. Ultralarge multimers of von Willebrand factor form spontaneous high-strength bonds with the platelet glycoprotein Ib-IX complex: studies using optical tweezers. Blood 2002;99(11):3971-3977

28 Goubran HA, Kotb RR, Stakiw J, Emara ME, Burnouf T. Regulation of tumor growth and metastasis: the role of tumor microenvironment. Cancer Growth Metastasis 2014;7:9-18

29 Wagner DD, Frenette PS. The vessel wall and its interactions. Blood 2008;111(11):5271-5281

30 Terraube V, Marx I, Denis CV. Role of von Willebrand factor in tumor metastasis. Thromb Res 2007;120(Suppl 2):S64-S70

31 Bauer AT, Suckau J, Frank K, et al. von Willebrand factor fibers promote cancer-associated platelet aggregation in malignant melanoma of mice and humans. Blood 2015;125(20):3153-3163

32 Wang WS, Lin JK, Lin TC, et al. Plasma von Willebrand factor level as a prognostic indicator of patients with metastatic colorectal carcinoma. World J Gastroenterol 2005;11(14):2166-2170

33 Zietek Z, Iwan-Zietek I, Paczulski R, Kotschy M, Wolski Z. von Willebrand factor antigen in blood plasma of patients with urinary bladder carcinoma. Thromb Res 1996;83(05):399-402

34 Oleksowicz L, Dutcher JP, Deleon-Fernandez M, Paietta E, Etkind P. Human breast carcinoma cells synthesize a protein immunorelated to platelet glycoprotein-Ib alpha with different functional properties. J Lab Clin Med 1997;129(03):337-346

35 Oleksowicz L, Dutcher JP, DeLeon-Fernandez M, Etkind P. A GPIb alpha-related protein is expressed by fresh human breast carcinoma tissue and is regulated by a PKC-sensitive mechanism. Exp Cell Res 1997;237(01):110-117

36 Verheul HM, Hoekman K, Luykx-de Bakker S, et al. Platelet: transporter of vascular endothelial growth factor. Clin Cancer Res 1997;3(12, Pt 1):2187-2190 